\title{
Construction of Education Management Information System Based on Cloud Computing
}

\author{
Zhao Lijun \\ Xi'an Siyuan University, Xi'an 710038, China \\ Zhaolijun@126.com
}

Keywords: cloud computing; education management information system; education information

\begin{abstract}
Based on the concept and characteristics of cloud computing, combining with the problems existing in the construction of education management information system, starting from the cost-saving and cycle education information construction to improve the degree of resource sharing, and security, the education management information system is constructed based on cloud computing.
\end{abstract}

\section{Introduction}

More and more attention has been paid to the construction of university digital campus. As the basic management information system of digital campus construction [1], it also faces many problems and challenges while developing rapidly. For example, the infrastructure resources to provide services are scattered, difficult to allocate, serious repeated purchases and low utilization rate. Due to the different development environments and technical standards of various management platforms, the sharing degree is poor, the update speed is slow, and there is no unified management infrastructure, so it is urgent to improve the management information system. Based on the concept of cloud computing, this paper constructs and maintains the management information system of colleges and universities. The new idea of protection was put forward.

\section{Concept of Cloud Computing}

Cloud computing is a on-demand, convenient and scalable information delivery service based on the Internet, also known as cloud services [2]. The "cloud" in cloud computing can be said to be a freely sized and unconstrained cloud, which indirectly reflects the high transparency and scalability of cloud computing in information resource construction and service. The basic connotation of cloud computing can be from the perspective of system and technology, summarized as the following three parts: (1) Software as a service (SaaS: Software as a Service): refers to a variety of applications for the cloud user directly; PaaS: Platform as a Service: mainly including the development and operation of the Platform; The IaaS Infrastructure as a Service: it mainly includes the construction of storage, computing and network equipment. The core of cloud computing is distributed computing, virtualization, and high-speed networks.

\section{Current Situation of Education Information System}

The management information system of colleges and universities is involved in teaching and research, administrative office, personnel management [3], life service and other aspects. While improving the efficiency of the department, the following problems are also generated:

\subsection{Data storage and management pressure}

The scientific research and teaching management information system in colleges and universities has produced tens of thousands of tons of data, which brings great pressure to the management and storage of database [4]. For example, in the course selection node system, the system server is often unable to withstand high traffic, leading to the phenomenon of course selection congestion, which 
affects the orderly teaching work [5].

\subsection{The low software and hardware resources utilization rate and sharing degree}

Because the development environment and the technical standards adopted are different, the management information system has great limitations in compatibility and collaboration. The differences in the platform result in the sharing of hardware and software resources between departments. The repeated procurement of hardware and software equipment and related resources by various departments caused unnecessary waste. Due to the development of information technology and the change of demand, the original management platform faces the risk of elimination.

\subsection{Information island phenomenon}

Due to the lack of unified planning and the single function between the systems, the centralized architecture of the database makes it impossible to share data [6]. The subordinate unit cannot achieve secondary development according to its own needs, which leads to the low value and efficiency of data use and affects the business cooperation between different departments, such as the island where information is scattered.

\section{Advantages of Cloud Computing in the Construction of Education Management Information System}

High scalability, high reliability, sharing and virtualization of cloud computing can solve the disadvantages of university management information system scientifically and efficiently, and its advantages are reflected in the following aspects [7]:

\subsection{Save hardware and software costs}

Cloud computing saves the software and hardware cost of college information construction. The average physical server has only 20 to 25 percent memory utilization and most of its time and resources are idle. If time, traffic and bandwidth are used to rent the cloud server, it will greatly save hardware and software costs and human resources.

\subsection{Efficient and secure data storage service}

Cloud computing has strong data processing and management ability, which provide the cloud storage service can use the Internet, spread all regions of the different types of storage devices together to work together, through the common external applications provide data acquisition, storage, and business access, and other functions. All data is managed uniformly through cloud server, greatly reducing the workload of maintenance personnel. At the same time, cloud computing service providers provide professional maintenance management services to ensure data security.

\subsection{Eliminate information isolation and enhance communication and sharing among departments}

The management information system is built based on the cloud platform, and the data between departments are uniformly managed and extracted from the cloud, avoiding the ununified and independent data between departments. Make reasonable planning for the whole information system, so as to improve the problems such as too long information transfer time between departments, overlapping and duplication of work, etc. At the same time, it is possible to maximize resource sharing and collaborative work among various departments in universities under the network environment by utilizing the strong management mechanism, automatic deployment and high virtualization of cloud computing.

\section{Cloud Platform Module Design of Education Management Information System}

The cloud platform of the university management information system should not only take into 
account the actual demand, but also the expansion and high scalability of the entire cloud platform. The requirements of the functional modules of the platform are as follows:

The first is to be able to dynamically migrate and load balanced computing resource pools. For base layer resources need to be smart supply and recycling, need at least two servers, which is used to manage the resources of the base layer and data storage server as cloud computing server computing nodes, node, of course, can also according to the actual need to expand; Followed by cloud storage resource pool, main function is through the study of the virtualization of base layer facilities management, and scientific computing the optimal capacity of base layer resources, that is to calculate how much can the service information management system; Third, it can solve the existing network scale limitation, traffic congestion, high cost and other problems.

\section{System Data Encryption and Decryption}

\subsection{Encryption of data}

Before uploading the data file, the user first USES the symmetric encryption algorithm to Encrypt the file, and then USES the Encrypt algorithm to Encrypt the obtained key again. The detailed process is as follows:

1) during the access, the depth of the random polynomial $q_{x}$ is set to $d_{x}=k_{x}-1$ for all nodes in the control structure A of the access, i.e., 1 less than the threshold value.

2 ) after the first step, select a random key from the root node R and set $q_{R}(0)=s$, while the other nodes $\mathrm{x}$ are $q_{x}(0)=q_{\text {parent }(x)}(\operatorname{index}(x))$

3) all leaf nodes are stored in the collection to encrypt the symmetric key for accessing A node $\mathrm{Y}$ in structure A:

$$
\begin{aligned}
& C T=\left(C=k e(g, g)^{\alpha s}, C^{\prime}=g^{\beta s},\right. \\
& \left.\forall y \in Y: C_{y}=g^{q_{y}(0)}, C_{y}^{\prime}=H(\operatorname{att}(y))^{q_{y}^{(0) v_{y}}}\right)
\end{aligned}
$$

Description: in the above calculation, parent $(x)$ refers to the parent node of the returned node, $\operatorname{index}(x)$ represents the number of the node, and $\operatorname{att}(y)$ represents the attribute of the node.

\subsection{Decryption of data}

After capturing the user in the cloud server data file, HMAC is first used to verified the integrity of the data file, confirm that data are complete, use Decrypt decryption decryption algorithm, and only when the user attributes is required to access control structure, in order to success the key encrypted cryptograph.

When the node is a leaf node, set $i=\operatorname{att}(y)$, and then calculate the leaf node using the DecryptNode $\left(\mathrm{CT}_{k}, S K_{u}, x\right)$ algorithm for each leaf node:

$$
\left\{\begin{array}{l}
\frac{e\left(K_{x}^{\prime}, C_{x}\right)}{e\left(K_{x}^{\prime \prime}, C_{x}\right)}=\frac{e\left(g^{\alpha t} \cdot H(x)^{r_{x} v_{x}}, g^{q_{x}(0)}\right)}{e\left(g^{r_{x}}, H(i)^{q_{x}(0) v_{y}}\right)}=e(g, g)^{\alpha t q_{x}(0)}, x \in A \\
\perp, x \notin A
\end{array}\right.
$$

The interpolation nodes of $\mathrm{e}(g, g)^{r q_{x}(0)}$ Lagrange polynomials are used to calculate non-leaf nodes: 


$$
\begin{aligned}
& F_{x}=\prod_{z \in S_{x}} F_{z}^{\Delta i, S_{x(0)}^{\prime}}\left(i=\operatorname{index}(z), s_{x}^{\prime}=\left\{\operatorname{index}(z): z \in S_{x}\right\}\right) \\
& =\prod_{z \in S_{x}}\left(e(g, g)^{\alpha t \cdot q_{z}(0)}\right)^{\Delta i, S_{x(0)}^{\prime}} \\
& =\prod_{z \in S_{x}} e(g, g)^{\alpha t \cdot q(i) \cdot \Delta i, S_{x(0)}^{\prime}} \\
& =e(g, g)^{\alpha t \cdot q_{x}(0)}
\end{aligned}
$$

Pending decrypts the encrypted key

Set $\mathrm{A}=e(g, g)^{\alpha t q_{R}(0)}=e(g, g)^{\alpha t s}$, and you get

$$
K=C /\left(\mathrm{e}\left(C^{\prime}, K\right) / A\right)
$$

After the user gets the symmetric encryption key, the cipher text can be decrypted to obtain the plaintext M.

\section{The Overall Architecture of Cloud Platform of Education Management Information System}

The management information system cloud platform is divided into three levels.

\subsection{Infrastructure layer (IaaS)}

The first layer is the lowest level infrastructure layer (IaaS), which is mainly divided into physical hardware sub-layer and virtual hardware sub-layer. The IaaS layer mainly integrates and distributes software and hardware resources through virtualization technology to avoid the impact of differences, so as to form a resource pool that can effectively monitor and arrange resources. Mainly including network resources, server resources, hardware resources, and corresponding database backup, computing services.

\subsection{Platform Service layer (PaaS)}

The second layer is the platform service layer (PaaS) between the infrastructure layer and the application layer. Mainly by integrating various service middleware, guided by the demands of management, construct of base layer resources on-demand dynamic allocation development platform, based on user needs to provide a series of authentication management, event log, content note, data transfer and other services.

\subsection{Software service layer (SaaS)}

The third layer is the software services layer (SaaS) at the top level for end users. The main function is to allocate resources dynamically and intelligently, and then make quick response and support to various service requests. Provide "one-stop" service for various management information systems integrated on the campus based on cloud platform, such as administrative office system (OA), educational information management system, and logistics information management system.

\section{Conclusion}

Cloud computing is a new concept, the use of the concept and connotation can offer good service for all departments of colleges and universities management support, and all kinds of hardware and software resources to conduct a comprehensive integration of campus and dynamic allocation, and on this basis, for all the teachers and students scientific research and teaching to provide quality information services and management platform. 


\section{References}

[1] Chen Z, Han F, Cao J, et al. Cloud computing-based forensic analysis for collaborative network security management system[J]. Tsinghua Science and Technology, 2013, 18(1):40-50.

[2] Wan L, Han G, Shu L, et al. Distributed Parameter Estimation for Mobile Wireless Sensor Network Based on Cloud Computing in Battlefield Surveillance System[J]. IEEE Access, 2015, 3:1729-1739.

[3] Nguyen T D, Nguyen D T, Cao T H. Acceptance and Use of Information System: E-Learning Based on Cloud Computing in Vietnam[J]. Lecture Notes in Computer Science, 2014, 8407:139-149.

[4] Riahi G. E-learning Systems Based on Cloud Computing: A Review[J]. Proscenia Computer Science, 2015, 62:352-359.

[5] Caminero A, Ros S, Hernandez R, et al. Virtual remote laboratories management System (TUTORES): Using cloud computing to acquire university practical skills[J]. IEEE Transactions on Learning Technologies, 2016, 9(2):133-145.

[6] Wang Y, Wang L, Liu H, et al. Large-Scale Clinical Data Management and Analysis System Based on Cloud Computing[J]. Lecture Notes in Electrical Engineering, 2014, 269:1575-1583.

[7] Fang S, Zhu Y, Xu L, et al. An integrated system for land resources supervision based on the IoT and cloud computing[J]. Enterprise Information Systems, 2015, 11(1):105-121. 